\title{
Informationen aus dem Sekretariat
}

Marianne Roth | Ursula Enggist | Claudia Menolfi

\section{EXKLUSIV FÜR ASP-MITGLIEDER}

\section{Protokoll der ordentlichen Mitgliederversammlung der ASP vom 24. März 2018}

Der Versand des Protokolls in Deutsch, Französisch und Italienisch erfolgte per E-Mail an unsere Mitglieder. Eine Papierversion kann im Sekretariat bestellt werden oder ist im Mitgliederbereich der Website abrufbar.

Die nächste Mitgliederversammlung findet am 23. März 2019 in Zürich statt.

\section{IV-Vertrag}

Psychotherapien für Kinder und Minderjährige, Schwierigkeiten bei der Geburt und zur beruflichen Eingliederung können unter bestimmten Voraussetzungen über die Integrierte Versorgung (IV) abgerechnet werden. Eidgenössisch anerkannte ASP-Mitglieder mit Praxisbewilligung haben auf Antrag die Möglichkeit, dem Vertrag zwischen dem Bundesamt für Sozialversicherung (BSV) und der ASP beizutreten. Informationen liefert unsere Website oder die ASP-Geschäftsstelle, die auch die Anträge entgegennimmt.

\section{Abrechnungsformulare}

Für die Rechnungsstellung des Honorars an ihre Patientinnen und Patienten können Mitglieder im Sekretariat die praktische, beschreibbare PDF-Vorlage bestellen oder im geschützten Mitgliederbereich der Website herunterladen.

\section{Leistungen und Tarife}

Das Merkblatt mit den Richtlinien der ASP für Leistungen und Tarife ist im Mitgliederbereich unserer Website unter der Rubrik «Merkblätter» abrufbar.

\section{Krankenkassenliste}

Wissen Sie, welche Krankenkasse welchen Betrag für Psychotherapie vergütet? Verlangen Sie im Sekretariat die Krankenkassenliste oder informieren Sie sich auf unserer Website im Bereich «Downloads».

\section{ASP-Mitglieder sind \\ Botschafter und Botschafterinnen des Psychotherapieberufes}

Nutzen Sie die Gelegenheit, Ihre Patientinnen und Patienten über Ihre eidgenössische Anerkennung und den Titelschutz zu informieren. Klären Sie sie über ihre Rechte und Pflichten auf, über die Schweigepflicht und den Schutz, den sie als Ihre Patientin oder Ihr Patient geniessen.

\section{NEU: Verschlüsselte HIN-E-Mail-Adresse}

Sichern Sie sich zu Sonderkonditionen für ASP-Mitglieder Ihre persönliche verschlüsselte HIN-E-Mail-Adresse. Damit sorgen Sie für einen geschützten Transfer Ihrer sensiblen elektronischen Daten.

\section{Stelleninserate und Praxisräume}

Besuchen Sie die Rubrik «Aktuell» auf unserer Website. Neben aktuellen Informationen und Verbandsnachrichten finden Sie Ausschreibungen für offene Stellen und Praxisräume. Haben Sie ein eigenes Angebot oder wünschen Sie nähere Auskünfte? Kontaktieren Sie das Sekretariat für weitere Angaben zur Platzierung auf unserer Website.

\section{Eintrag unserer Mitglieder im PsyReg}

Alle unsere ordentlichen Mitglieder sind im PsyReg als eidgenössisch anerkannte Psychotherapeutinnen und Psychotherapeuten eingetragen. Neu aufgenommene Mitglieder werden automatisch dem Bundesamt für Gesundheit (BAG) zur Aufnahme ins Register gemeldet.

Änderungswünsche Ihres Eintrags müssen Sie direkt beim BAG platzieren.

Für den gültigen Eintrag der kantonalen Berufsausübungsbewilligungen im PsyReg sind die kantonalen Gesundheitsdirektionen zuständig.

\section{Fortbildungspflicht für Mitglieder}

Psychotherapeutinnen und Psychotherapeuten sind gesetzlich verpflichtet, laufend Fortbildungen zu besuchen. Diese dienen der wissenschaftlichen Fortbildung, dem Theoriestudium sowie der Erweiterung und Vertiefung praktischer Kompetenzen. Unsere Website liefert weitere Informationen und unser umfassendes Fortbildungsprogramm unter der Rubrik «Weiterbildung \& Fortbildung».

\section{Psychotherapierelevante Publikationen und Informationen}

Im Mitgliederbereich auf unserer Website finden Sie die Publikationen, die für Sie als ASP-Mitglied bindend sind. Neben Reglementen nehmen Merkblätter Bezug auf diverse Fragen zu spezifischen Bereichen des Psychotherapieberufes. Nutzen Sie unser Sekretariat als Informations- und Auskunftsstelle.

\section{NEU: Archivieren Ihrer Dokumentation}

Planen Sie, sich aus dem aktiven Berufsleben zurückzuziehen und möchten Ihre Akten sicher archivieren? Möchten Sie Ihre gesammelte Dokumentation im Sinne einer Nachlassregelung sicher aufbewahrt wissen?

Die ASP kann ihren Mitgliedern exklusiv eine kostengünstige und sichere Lösung anbieten. Weitere Auskünfte erhalten Sie in unserem Sekretariat. 


\section{VERSICHERUNGEN}

\section{All-Inclusive-Paket}

Dank dem Kollektivvertrag mit unserer Versicherungsagentur, Swiss Quality Broker AG, sind wir in der Lage, unseren Mitgliedern ein exklusives Paket an günstigen Versicherungsleistungen anzubieten, das folgende Angebote beinhaltet:

- Krankenkasse

- Berufshaftpflichtversicherung

- Rechtsschutzversicherung

- Geschäftssachversicherung

- Kranken- und Unfalltaggeldversicherung

- Erwerbsausfallversicherung

ASP Mitglieder können zudem bei der ZURICH Versicherung in den Genuss von günstigeren Prämien für ihre Autoversicherung, Motorradversicherung, Hausrat-, Privathaftpflicht- und Gebäudeversicherung kommen.

Informieren Sie sich über die Details im geschützten Mitgliederbereich auf unserer Website.

\section{Weitere Empfehlung:}

\section{- Pensionskasse (Zweite Säule)}

Die Pro Medico Stiftung Zürich bietet als Verbandsvorsorge der zweiten Säule den ASP-Mitgliedern die Möglichkeit einer flexiblen beruflichen Vorsorge. Es handelt sich um finanziell attraktive Angebote zur individuellen Altersvorsorge für selbstständig Erwerbende und deren Personal.

\section{ALLGEMEINE INFORMATIONEN}

\section{www.psychotherapie.ch}

Unsere Website enthält sämtliche relevanten Informationen für unsere Mitglieder in einem geschützten Bereich mit Passwortzugang. Die Termine der nächsten Mitgliederversammlung, Kolloquien für die Charta-Konferenz, Versicherungslösungen etc. befinden sich ebenfalls unter dieser Rubrik. Wir empfehlen einen regelmässigen Besuch.

Neben Angaben zu unserer Organisation und über Psychotherapie allgemein enthält die Website Informationen für die breite Öffentlichkeit zu den Themen:

- Berufspolitik \& Berufsentwicklung

- Weiterbildung \& Fortbildung

- Wissenschaft \& Forschung.

Ausschreibungen über aktuelle Veranstaltungen, Fortbildungen, Tagungen etc. befinden sich auf unserer Website unter der Rubrik «Agenda».

\section{Aktuelles}

Informieren Sie sich beispielsweise zum Stand des Anordnungsmodells sowie über weitere brennende Themen auf unserer Website unter der Rubrik «Aktuell».
Haben Sie einen Wunsch oder eine Anregung? Wenden Sie sich damit an unsere Geschäftsstelle.

\section{PUBLIKATIONEN}

\section{Zeitschrift à jour! - Psychotherapie-Berufsentwicklung}

Die Zeitschrift à jour! ist das zweisprachige (Deutsch und Französisch) Informationsorgan der ASP mit berufspolitischen und fachlichen Inhalten. Sie versteht sich als Bindeglied zwischen uns als Berufsverband und unseren Mitgliedern sowie allen an Psychotherapie interessierten Leserinnen und Lesern.

\section{Zeitschrift Psychotherapie-Wissenschaft}

Die Zeitschrift Psychotherapie-Wissenschaft steht im Dienste der Entwicklung der Psychotherapie. Beiträge zu Praxis und Forschung fördern den interdisziplinären Austausch über grundlegende Fragen wie Indikation, Methodik, Wirksamkeit.

Beide Ausgaben erscheinen zweimal jährlich. Übrigens sind die Beiträge beider Zeitschriften im Internet als digitale Version aufrufbar unter: www.psychotherapie-wissenschaft.info

\section{Anzeigen}

Nutzen Sie die Möglichkeit zur Publikation Ihrer Bekanntmachungen oder Anzeigen in unseren Zeitschriften. Zielpublikum der publizierten Beiträge sind praktizierende Psychotherapeutinnen und Psychotherapeuten sowie Fachleute aus Wissenschaft, Forschung und Praxis. Erkundigen Sie sich an der Geschäftsstelle über Publikationsmöglichkeiten, Preise und Erscheinungsweisen.

\section{Newsletter}

Unser Newsletter hält Sie über aktuelle Aktivitäten des Verbands sowie berufspolitische Entwicklungen auf dem Laufenden. Er erscheint viermal jährlich.

\section{ORGANISATORISCHES}

\section{Medien}

Die Geschäftsstelle nimmt Anfragen von Medienschaffenden gerne entgegen. Unser Verband vereinigt versierte Praktikerinnen und Praktiker zu verschiedenen - auch tagesaktuellen - Themen und Psychotherapiemethoden.

\section{ASP-Vorstandsmitglieder}

- Gabriela Rüttimann, Präsidentin

- Veronica Defièbre, Vizepräsidentin, Leitung Charta-Konferenz

- Nicola Gianinazzi, Delegierter italienische Schweiz

- Peter Schulthess, leitender Redakteur à jour! und Psychotherapie-Wissenschaft 


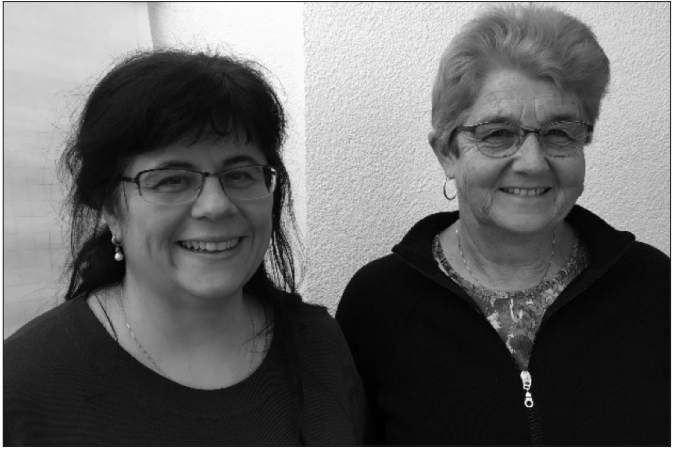

(v. I.): Claudia Menolfi und Ursula Enggist

\section{Geschäftsstelle}

- Marianne Roth, Geschäftsleiterin

- Ursula Enggist, Sekretariat

- Claudia Menolfi, Sekretariat

Kontakt:

Telefon: 0442689300

marianne.roth@psychotherapie.ch ursula.enggist@psychotherapie.ch claudia.menolfi@psychotherapie.ch

\section{Aktuelles}

aus der italienischen Schweiz

Psychologie, Biomedizin und neurowissenschaftliches Supercomputing

begegnen sich

Nicola Gianinazzi

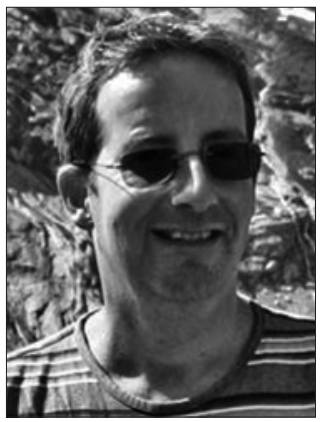

Im Einvernehmen mit dem Ausschuss erhält meine Rolle als Kommunikationsantenne zwischen der italienischsprachigen Schweiz und den anderen Teilen der Eidgenossenschaft zunehmend Gewicht.

Als Delegierter arbeite ich zudem bei der Vorbereitung und Betreuung der Endphase der definitiven Akkreditierung intensiv mit der IRG zusammen. Insbesondere haben wir auch die Begleitung der von der AAQ benannten Experten auf ihrem Besuch bei der IRG vereinbart.

$\mathrm{Ab}$ diesem Jahr werden die italienische Schweiz und das italienische Panorama - durch die Aufnahme in die Redaktion der Psychotherapie-Wissenschaft - in unserem thematischen Magazin präsenter und sichtbarer.

Schliesslich wurde die Arbeit der Kontaktaufnahme mit Hochschullehrern verschiedener Universitäten und Fakultäten im Tessin weiter vertieft. Diese interessante Tätigkeit der «Public Relations» ist nützlich und notwendig, um sowohl die Ausbildungsvorschläge, die Beiträge unserer Zeitschriften als auch die Perspektiven der Entwicklung von Lehre und Forschung zu bereichern. In diesem Zusammenhang wird auch die redaktionelle Zusammenarbeit mit der Zeitschrift Psicoterapia e scienze umane (www.psicoterapia escienzeumane.it) verstärkt, deren Co-Direktor Paolo Migone ist.

\section{Das Tessiner Panorama}

Ab Herbst 2020 beginnen die Studiengänge der USI an der Fakultät für Biomedizinische Wissenschaften, die in Lugano in Zusammenarbeit mit den Universitäten Basel und Zürich sowie der ETH Zürich gegründet wurde. Sie wird in Synergie mit anderen Fakultäten der USI in Bereichen wie Gesundheitskommunikation, Gesundheitsmanagement und Gesundheitswirtschaft, der medizinischen Anwendung der Computational Science arbeiten und sollte auch die Psychiatrie einschliessen.

Besonders nahe an unserem psychotherapeutischen Bereich sind diejenigen Disziplinen mögliche Weiterbildungskurse für uns Psychotherapeuten -, die sich auf die folgenden Themen im Bereich Beziehung und Kommunikation in der Gesundheit beziehen (gemäss dem, was ich persönlich als das «Neue modulare Modell der Psychotherapie-Wissenschaften» bezeichnen würde):

Rhetorik, Argumentation und Überzeugungs$\mathrm{kraft} /$ Risikokommunikation/Gesundheitsför- 